\title{
Summation of Series of Positive Terms by Condensation Transformations*
}

\author{
By James W. Daniel**
}

\begin{abstract}
The condensation transformation, which maps series of positive terms into more conveniently summed alternating series, each term $v_{j}$ of which is itself an infinite series, is discussed with examples. It is shown that for a large class of extremely slowly convergent series (essentially those dominated by the "logarithmic scale") the series defining the terms $v_{j}$ are more easily summed than the original and may in fact be transformed further if desired. Numerical examples reveal the power of the method.
\end{abstract}

I. Introduction. When faced with the necessity of calculating the sum of an infinite series, an ingenious analyst can often obtain satisfactory results by using analytical techniques such as the Euler-Maclaurin sum formula, the calculus of residues, estimation by integrals, and so forth; sometimes, however, even the most wily must resort merely to adding up terms; probably aided by some acceleration technique.

If the series of interest is an alternating one, the classical method of Euler or the more recent $\epsilon$-algorithm usually converges fairly rapidly; these methods are widely used and easily programmed for a computer [3], [6]. If the series consists of positive terms, however, one usually uses some other suitable transformation such as the $\rho$-algorithm, Romberg extrapolation, and so on, to improve the convergence of the sequence of partial sums. Recently A. van Wijngaarden [4] has suggested a simple linear transformation of the original series into an alternating series, a technique which appears to be of great value, particularly for very slowly convergent series [1]; it will be seen that the advantage of this technique lies in its ability to transform series repeatedly until one is obtained for which simple summation or application of an aforementioned acceleration method will be successful. This paper describes the method, examines some theoretical aspects, and shows with numerical examples how it can be used in practice.

II. The Basic Transformation. Let $s=\sum_{i=1}^{\infty} a_{i}$ be the series of positive terms we seek to evaluate. Formally, we define $v_{j}=\sum_{i=1}^{\infty} b_{j, i}, b_{j, i}=2^{i-1} a_{j 2} i-1$, and see that $\sum_{j=1}^{\infty}(-1)^{i+1} v_{j}=\sum_{i=1}^{\infty} a_{i}=s$. Thus $s$ is obtained as the sum of an alternating series of terms $v_{j}$ each of which is defined as an infinite series, hopefully more readily summed than the original. For example, suppose $a_{i}=i^{-c}, c>1$. Then we have

$$
v_{j}=\sum_{i=1}^{\infty} 2^{i-1}\left(j 2^{i-1}\right)^{-c}=j^{-c} \sum_{i=1}^{\infty} 2^{(1-c)(i-1)}=j^{-c}\left(1-2^{1-c}\right)^{-1},
$$

Received April 10, 1968, revised July 29, 1968.

* This research was initiated with the support of the Office of Naval Research.

** Sponsored by the Mathematics Research Center, United States Army, Madison, Wisconsin, under Contract No. DA-31-124-ARO-D-462. 
and the series for each $v_{j}$ exhibits geometric convergence, an improvement over the original series. Summing the alternating series yields the classical result

$$
\sum_{i=1}^{\infty} i^{-c}=\left(1-2^{1-c}\right)^{-1} \sum_{j=1}^{\infty}(-1)^{j+1} j^{-c} .
$$

Generally $v_{j}$ will not be summable in closed form, but it will be easier to sum than $s$.

Observing that $\sum_{i=1}^{N} a_{i}=\sum_{i=1}^{N}\left(v_{2 i-1}-v_{2 i}\right)-\sum_{i=N+1}^{2 N} v_{i}$, van Wijngaarden proved the following:

ThEOREM 1. $\sum_{i=1}^{\infty} a_{i}$ and $\sum_{j=1}^{\infty}(-1)^{i+1} v_{j}$ both exist and are equal if and only if

(a) $\sum_{i=1}^{\infty} a_{i}$ converges,

(b) $v_{j}$ is finite for all $j$,

(c) $\lim _{N \rightarrow \infty} \sum_{i=N+1}^{2 N} v_{i}=0$.

The simplest theorem that yields the above three conditions is

Theorem 2. Suppose that $a_{i}, i=1,2, \cdots$, is a monotone decreasing sequence; then (Cauchy's condensation theorem) $v_{1}$ is finite if and only if $\sum_{i=1}^{\infty} a_{i}<\infty$. If $v_{1}$ is finite then all $v_{j}$ are finite and $\sum_{j=1}^{\infty}(-1)^{i+1} v_{j}=\sum_{i=1}^{\infty} a_{i}$.

Proof. The first statement is merely Cauchy's condensation theorem [2]; for the second we use Theorem 1.

$$
\sum_{i=1}^{N+1} b_{j, i}=a_{j}+2 \sum_{i=2}^{N+1} 2^{i-2} a_{j 2} i_{1} \leqq a_{j}+2 \sum_{i=2}^{2^{N}} a_{j i} .
$$

Now $a_{j i} \leqq j^{-1} \sum_{k=1}^{j} a_{j(i-1)+k}$; inserting this in the preceding yields

$$
\sum_{i=1}^{N+1} b_{j, i} \leqq a_{j}+\frac{2}{j} T_{j}
$$

where $T_{j} \equiv \sum_{i=j+1}^{\infty} a_{i}$ is the tail of a convergent series. Thus $v_{j} \leqq a_{j}+2 T_{j} / j<\infty$, and hence

$$
\sum_{i=N+1}^{2 N} v_{i} \leqq T_{N}+2 \sum_{i=N+1}^{2 N} \frac{T_{i}}{i} \leqq T_{N}+2 \sum_{i=N+1}^{2 N} \frac{T_{N}}{N} \leqq 3 T_{N} .
$$

Since $\lim _{N \rightarrow \infty} T_{N}=0$, the conditions of Theorem 1 are met. Q.E.D.

Corollary. Suppose $a_{i} \leqq A_{i}$ where $A_{i}$ is a monotone decreasing sequence such that $\sum_{i=1}^{\infty} A_{i}<\infty$. Then $v_{j}$ exists for all $j$ and $\sum_{j=1}^{\infty}(-1)^{j+1} v_{j}=\sum_{i=1}^{\infty} a_{i}$ both converge.

If $a_{i}$ is not dominated by a monotone sequence, only nearly trivial conditions are known which guarantee the conditions of Theorem 1; the following is typical.

Proposition 1. Suppose $\sum_{i=1}^{\infty} a_{i}<\infty$ and a sequence of positive numbers $L_{j}$ exists such that $2^{i-1} a_{j 2^{2}-1} \leqq L_{j} a_{i}$ for all $i \geqq 1$ and all odd $j \geqq 1$. Then $v_{j}$ exists for all $j$; if $\lim _{N \rightarrow \infty} \sum_{i=N+1}^{2 N} L_{j}=0$, then $\sum_{j=1}^{\infty}(-1)^{i+1} v_{j}=\sum_{i=1}^{\infty} a_{i}$.

III. Nature of the Transformation. Clearly the transformation from the series in $a_{i}$ to that in $v_{j}$ is a linear one; it is not, however, regular in the sense of preserving convergence and limit for arbitrary positive series. Perhaps the transformation should be called antiregular since, as the following shows, it is divergence preserving.

Theorem 3. If $\sum_{i=1}^{\infty} a_{i}=\infty$, then either $v_{j}=\infty$ for some $j$ or $\sum_{j=1}^{\infty}(-1)^{i+1} v_{j}$ diverges to $+\infty$.

Proof. Suppose $v_{j}<\infty$ for all $j$. Since we have 


$$
\sum_{i=1}^{N} a_{i}=\sum_{i=1}^{N}\left(v_{2 i-1}-v_{2 i}\right)-\sum_{i=N+1}^{2 N} v_{i} \leqq \sum_{i=1}^{N}\left(v_{2 i-1}-v_{2 i}\right),
$$

the last series diverges. Q.E.D.

All other combinations of convergence and divergence can occur, however, as the next examples show.

(1) $\sum_{i=1}^{\infty} a_{i}<\infty$, but some $v_{j}=\infty$ :

$$
a_{2^{n}}=\frac{1}{2^{n}}, \quad n=0,1, \cdots, a_{i}=\frac{1}{i^{2}} \text { otherwise } .
$$

(2) $\sum_{i=1}^{\infty} a_{i}=\infty, \sum_{j=1}^{\infty}(-1)^{j+1} v_{j}$ diverges, $v_{j}<\infty$ for all $j$ :

$$
a_{j 2^{n}}=\frac{1}{j \cdot 4^{n}}, \quad n=0,1, \cdots, j \text { odd } .
$$

Then, $v_{j 2^{m}}=2 / j 4^{m}, m=0,1, \cdots, j$ odd. Here

$$
\lim _{k \rightarrow \infty} v_{k}=0, v_{j 2^{m}-1}-v_{j 2^{m}}=\frac{2}{j 2^{m}-1}-\frac{\llcorner}{j 4^{m}} \geqq 0 .
$$

For $m>0$ the sum over odd $j$ of these terms diverges so $\sum_{j=1}^{\infty}(-1)^{j+1} v_{j}$ diverges.

(3) $\sum_{i=1}^{\infty} a_{i}<\infty, \sum_{j=1}^{\infty}(-1)^{j+1} v_{j}$ divergent:

$$
a_{j 2^{n}}=\frac{1}{2^{n}\left(1+1 / j^{\alpha}\right)^{n} j^{2}}, \quad \alpha \geqq 2, \quad j \text { odd } .
$$

Here $v_{j 2^{m}}=j^{\alpha-2} / 2^{m}\left(1+1 / j^{\alpha}\right)^{m-1}$, so $\lim _{k \rightarrow \infty} v_{k}$ does not exist.

(4) $\infty>\sum_{i=1}^{\infty} a_{i} \neq \sum_{j=1}^{\infty}(-1)^{j+1} v_{j}<\infty$ :

$$
a_{j 2^{n}}=\frac{1}{2^{n}(1+1 / j)^{n} j^{2}}, \quad j \text { odd }
$$

Then

$$
\begin{aligned}
v_{j 2^{m}}= & \frac{1}{j 2^{m}(1+1 / j)^{m-1}}, \lim _{k \rightarrow \infty} v_{k}=0, \quad v_{j 2^{m}-1}-v_{j 2^{m}}=\frac{j 2^{m}}{\left(j 2^{m}-1\right)^{2}} \\
& -\frac{1}{j 2^{m}(1+1 / j)^{m-1}}=\frac{1}{j 2^{m}-2+1 / j 2^{m}}-\frac{1}{j 2^{m}(1+1 / j)^{m-1}} \geqq 0 .
\end{aligned}
$$

By adding and subtracting we compare the series composed of these last differences with the series

$$
\frac{1}{j 2^{m}-2}-\frac{1}{j 2^{m}}=\frac{2}{j 2^{m}\left(j 2^{m}-2\right)}
$$

and it follows easily that $\sum_{j=1}^{\infty}(-1)^{j+1} v_{j}$ converges. Finally,

$$
\begin{aligned}
\sum_{i=1}^{2 N} a_{i}= & \sum_{i=1}^{2 N}\left(v_{2 i-1}-v_{2 i}\right)-\sum_{i=2 N+1}^{4 N} v_{i} \leqq \sum_{i=1}^{2 N}\left(v_{2 i-1}-v_{2 i}\right)-v_{2 N+1} \\
& -v_{2 n+3}-\cdots-v_{4 N-1}=\sum_{i=1}^{2 N}\left(v_{2 i-1}-v_{2 i}\right)-\frac{2 N+2}{(2 N+1)^{2}} \\
& -\cdots-\frac{4 N}{(4 N-1)^{2}} \leqq \sum_{i=1}^{2 N}\left(v_{2 i-1}-v_{2 i}\right)-N \cdot \frac{4 N}{(4 N-1)^{2}} .
\end{aligned}
$$


Letting $N$ tend to infinity yields $\sum_{i=1}^{\infty} a_{i} \leqq \sum_{j=1}^{\infty}(-1)^{j+1} v_{j}-\frac{1}{4}$.

Since we hope to compute $v_{j}$ more easily than $s$, it is fortunate that for decreasing $a_{i}$ the series for $v_{j}$ converges faster than the original in the sense that $\sum_{i=N+1}^{\infty} b_{j, i}$ $\leqq j^{-2} \sum_{i=j 2^{N-1}+1}^{\infty} a_{i}$; therefore, to compute $v_{j}$ or $s$ to within a fixed absolute error, fewer terms are required for the former. It is not true in general that

$$
\lim _{N \rightarrow \infty} \sum_{i=N+1}^{\infty} b_{j, i} / \sum_{i=N+1}^{\infty} a_{i}=0,
$$

a common definition of faster convergence; the limit is zero however for the useful test series

$$
a_{i}(p, c)=1 /\left(i\left(\ln _{1} i\right)\left(\ln _{2} i\right) \cdots\left(\ln _{p-1} i\right)\left(\ln _{p}^{c} i\right)\right), \text { for } c>1,
$$

where $\ln _{1} x=\ln x, \ln _{p+1} x=\ln \left(\ln _{p} x\right)$. For these series in fact the series defining $v_{j}$ converges like $a_{i}(p-1, c)$.

IV. Recursive Condensation. For notational convenience, let $v^{(0)}$ be the set of $v_{j}$ obtained by applying the condensation transformations to the original series $s=\sum_{i=1}^{\infty} a_{i}$; for $n \geqq 1$, let $v^{(n)}$ refer to the set of $v_{j}$ obtained by applying the transformations to each series defining the elements of $v^{(n-1)}$. One would hope that elements of the successive sets $v^{(n)}$ would be increasingly easy to sum, thus allowing us to compute $s$ with few evaluations of $a_{i}$. The question arises as to the values of $n$ for which $v^{(n)}$ consists of finite elements.

Theorem 4. Let $a_{i} \leqq A_{i}$, where $A_{i}, i A_{i}, i(\ln i) A_{i}, \cdots, i(\ln i) \cdots\left(\ln _{p} i\right) A_{i}$ all decrease as $i$ increases. If $\sum_{i=1}^{\infty} A_{i}<\infty$, then for $n=0,1, \cdots, p+1$ the elements of $v^{(n)}$ exist and are finite, and the relevant alternating series converge to the appropriate sums.

Proof. By the Corollary to Theorem 2, the conclusion is valid for $n=0$. The series defining $v_{j}$ is

$$
\sum_{i=1}^{\infty} 2^{i-1} a_{j 2} i-1=\frac{1}{j} \sum_{i=1}^{\infty}\left(j 2^{i-1}\right) a_{j 2}{ }^{i-1} ;
$$

the terms in this series are dominated by

$$
j 2^{i-1} A_{j 2}{ }^{i-1} \leqq j 2^{i-1} A_{2}{ }^{i-1} \equiv j A_{i}{ }^{(1)} .
$$

Now we show that $A_{i}{ }^{(1)}$, and hence $j A_{i}{ }^{(1)}$, satisfies the assumptions of this theorem for $p$ replaced by $p-1$. For

$$
\begin{array}{r}
i(\ln i) \cdots\left(\ln _{m} i\right) A_{i}{ }^{(1)}=i(\ln i) \cdots\left(\ln _{m} i\right) 2^{i-1} A_{2} i-1=n(d \ln n+1) \ln (d \ln n+1) \\
\cdots \ln _{m}(d \ln n+1) A_{n}, \quad n=2^{i-1}, d=1 / \ln 2>1 .
\end{array}
$$

This in turn equals

$$
\begin{aligned}
\left(n(\ln n)(\ln \ln n) \cdots\left(\ln _{m+1} n\right) A_{n}\right) \cdot \frac{d \ln (n+1)}{\ln n} & \\
& \cdot \frac{\ln (d \ln (n+1))}{\ln \ln n} \ldots \frac{\ln (d \ln (n+1))}{\ln (\ln n)} .
\end{aligned}
$$

By assumption, the first factor in parentheses decreases for $m \leqq p-1$; for the other factors we let $f(x)=\ln _{m}(d x+1) / \ln _{m} x$ and see that the sign of $f^{\prime}(x)$ is that of 


$$
\begin{array}{r}
\ln _{m} x\left[\frac{1}{\ln _{m-1}(d x+1)} \cdot \frac{1}{\ln _{m-2}(d x+1)} \cdots \frac{1}{x+1 / d}\right]-\ln _{m}(d x+1) \\
\cdot\left[\frac{1}{\ln _{m-1} x} \cdot \cdots \frac{1}{x}\right]<0 .
\end{array}
$$

Therefore, defining $A_{i}{ }^{(n)}=2^{i-1} A_{2^{i-1}}^{(n-1)}$, we see that the assumptions of the theorem hold for $A_{i}{ }^{(n)}$ with $p$ replaced by $p-n$ for $n \leqq p$, and that the $A_{i}{ }^{(n)}$ dominate the elements of $v^{(n)}$. For $n=p$ we have $A_{i}{ }^{(p)}$ and $i A_{i}{ }^{(p)}$ decreasing, which implies the result of the theorem for $v^{(p)}$ and $v^{(p+1)}$. Q.E.D.

Corollary. If for some $p \geqq 0$ and $c>1$ we have $a_{i} \leqq A / i(\ln i) \cdots\left(\ln _{p}{ }^{c} i\right)$, then the $v^{(n)}$ exist for all $n \geqq 0$ and the relevant alternating series converge to the appropriate sums.

Since in practice most convergent positive series meet the requirements of Theorem 4, repeated use of the condensation transformations will generally be valid. In more pathological cases, the extension of Proposition 1 may be useful.

Proposition 2. If $\sum_{i=1}^{\infty} a_{i}<\infty$ and $2^{i-1} a_{j_{2}}{ }^{i-1} \leqq L_{j} a_{i}$, then the elements of $v^{(n)}$ exist for all $n \geqq 0$. If $\lim _{N \rightarrow \infty} \sum_{j=N+1}^{2 N} L_{j}=0$, then all the relevant alternating series converge to the appropriate sums.

Proof. We only need observe that

$$
2^{m-1} 2^{k 2^{m-1}-1} a_{j 2^{k 2^{m-1}-1}} \leqq L_{j} L_{k} a_{m} . \quad \text { Q.E.D. }
$$

V. Practical Summation. To devise a practical method of summation implementing condensation transformations, one faces the usual difficult questions of numerical analysis. How shall we define convergence so as to get answers in finite time? How shall we decide whether condensation is called for or whether the series can be summed in a more direct fashion? What should this direct fashion be? How should we sum the resulting alternating series? The list is far from complete.

Experience seems to indicate that even a rather naive set of answers to these questions will lead to a valuable summation method; of course the more refined the answers the more powerful the method. The real value of the overall approach lies in its ability to improve convergence repeatedly until a more straightforward method works. To give an indication of this ability in practice we report some simple numerical experiments based on an unsophisticated implementation of condensations; following the examples are some suggestions for a more sophisticated routine. Such routines are under development and will be reported elsewhere.

VI. Examples. Several examples were studied at the Mathematisch Centrum in Amsterdam and the Mathematics Research Center in Madison. In the program used, convergence was defined by van Wijngaarden's concept of incredulity [5]; convergence was assumed to occur whenever the approximate sum changed by less than a small number 'max zero' for 'tim' times in succession. If convergence would have been attained by direct addition of 'maxaddup' terms, the addition was performed; otherwise condensation was used, taking advantage of the relation $v_{2 j}=\frac{1}{2}\left(v_{j}-a_{j}\right)$. The resulting alternating series were summed by Euler's method as described in [3].

Consider $a_{i}=1 /(i+1) \ln ^{2}(i+1)$, the sum of whose series satisfies 2.109741 $\leqq S \leqq 2.109743$. For 'tim' $=1$ and ' $\max$ zero' $=10^{-3}, 10^{-4}, 10^{-6}$, we computed $2.1052,2.1046$, and 2.1090 requiring 181,392 , and 1021 evaluations of $a_{i}$ respec- 
tively; it is not clear how the error varies with 'max zero'. In all cases the program used elements from $v^{(0)}, v^{(1)}$, and $v^{(2)}$. Although this series can be summed easily by analytic techniques, the example shows the method's effectiveness on slowly convergent series; the remainder after directly adding 6300 terms is greater than .114 . An increase in 'tim' increases the accuracy, but not appreciably; for example, for 'tim' $=3$ and 'max zero' $=10^{-4}$ and $10^{-6}$ the sums are 2.1086 and 2.1090 .

Consider the series $\sum_{i=1}^{\infty}(1 / 1.001)^{i}=1000$, which is rather slowly convergent; the remainder after 3000 terms is greater than 50 . For 'tim' $=1$ and 'max zero' $=10^{-2}$ and $10^{-4}$, we obtained 999.95 and 999.9998 , in both cases using elements from $v^{(0)}$ and $v^{(1)}$.

For the series $\sum_{i=1}^{\infty} i^{-2}=\pi^{2} / 6=1.64493407$, with 'tim' $=1$ and ' $m a x$ zero' $=10^{-4}$ and $10^{-6}$, we obtained 1.6448 and 1.6449348 , using $v^{(0)}$ and $v^{(1)}$.

These examples serve to reveal both the power of the method and the weakness of its implementation; it seems clear that something other than direct summation should be used when condensation is not required. For example, we experimented briefly with the $\rho$-algorithm [6] substituted for direct summation and the repeated $\epsilon$-algorithm [5] for Euler summation. The results for the slowly convergent series of the first example above are striking; with 'tim' $=1$ and ' $\max$ zero' $=10^{-4}$ we obtained $s=2.1093$ with only 78 evaluations, a marked improvement in both accuracy and number of evaluations.

Clearly the ideal approach to an all-purpose summation routine would be a blend of such methods as the $\epsilon$-algorithm, $\rho$-algorithm, Romberg extrapolation, etc., with condensation used on extremely slowly convergent series like the logarithmic scale $a_{i}(p, c)$ to bring the convergence to a level which the other methods can handle. The crucial problem here of course is the automatic selection of the proper acceleration technique; at present we seem compelled to rely on a priori human judgement. Since the human usually knows something about his series to be summed, this situation is not unbearable.

Computer Sciences Department

The University of Wisconsin

Madison, Wisconsin 53706

1. National Physical Laboratory, Modern Computing Methods, 125, Philosophical Library, New York, 1961.

2. K. KNOPP, Theory and Application of Infinite Series, Hafner, New York, 1949.

3. P. NAUR (Editor), "Report on the algorithmic language ALGOL 60," Comm. ACM, v. 3, 1960, pp. 299-314. MR 24 \#B485.

4. A. van Wijngaarden, Cursus: Wetenschappelijk Rekenen B, "Process Analyse," Stichting Mathematisch Centrum, Amsterdam, 1965, pp. 51-60.

5. A. van WiJngaARDEN, "Numerical analysis as an independent science," Nordisk Tidskr. Informations-Behandling, v. 6, 1966, pp. 66-81. MR 34 \#2141.

6. P. WYNN, "A note on programming repeated application of the $\epsilon$-algorithm," Rev. Française Traitement Information Chiffres, v. 8, 1965, pp. 23-62. MR 31 \#5310.

7. P. WYNN, "On a Procrustean technique for the numerical transformation of slowly convergent sequences and series," Proc. Cambridge Philos. Soc., v. 52, 1956, pp. 663-671. MR 18, 478. 